\title{
Preferential $\mathrm{Cu}$ precipitation at extended defects in bcc Fe: An atomistic study
}

\author{
Yongfeng Zhang ${ }^{\mathrm{a}, *}$, Paul C. Millett ${ }^{\mathrm{b}}$, Michael R. Tonks ${ }^{\mathrm{a}}$, Xian-Ming Bai ${ }^{\mathrm{a}}$, S. Bulent Biner ${ }^{\mathrm{a}}$ \\ ${ }^{a}$ Fuels Modeling and Simulation Department, Idaho National Laboratory (INL), Idaho Falls, ID 83415, USA \\ ${ }^{b}$ Department of Mechanical Engineering, University of Arkansas, Fayetteville, AR 72701, USA
}

\begin{abstract}
As a starting point to understand $\mathrm{Cu}$ precipitation in RPV alloys, molecular dynamics and Metropolis Monte-Carlo simulations are carried out to study the effect of lattice defects on $\mathrm{Cu}$ precipitation by taking $\mathrm{Fe}-\mathrm{Cu}$ system as a model alloy. Molecular dynamics simulations show that owing to the high heat of mixing and positive size mismatch, $\mathrm{Cu}$ is attracted by vacancy type defects such as vacancies and voids, and tensile stress fields. In accordance, preferential precipitation of $\mathrm{Cu}$ is observed in Metropolis Monte-Carlo simulations at dislocations, prismatic loops and voids. The interaction of $\mathrm{Cu}$ with a stress field, e.g., that associated with a dislocation or a prismatic loop, is dominated by elastic effect and can be well described by the linear-elasticity theory. For prismatic loops, the attraction to $\mathrm{Cu}$ is found to be size-dependent with opposite trends displayed by vacancy and interstitial loops. The size-dependences can be explained by considering the stress fields produced by these loops. The current results will be useful for understanding the effect of neutron irradiation on $\mathrm{Cu}$ precipitation in reactor-pressure-vessel steels.
\end{abstract}

\section{Introduction}

The integrity of reactor pressure vessels (RPVs) is one of the primary safety factors that limit the service life of current light water reactors [1-3]. RPVs are made of low-alloy steels with $\mathrm{Mn}, \mathrm{Ni}$ and $\mathrm{Si}$ being the primary alloying elements, and $\mathrm{C}$ and $\mathrm{Cu}$ being either alloying elements or impurities. Due to the long service life and relatively high operating temperature, RPVs are subject to concurrent thermal ageing and fast neutron irradiation. During service, the microstructure of RPV steels evolves by precipitation of alloying elements, including Cu-rich-precipitates (CRPs) and Mn/Ni-Rich-Precipitates (MNPs) in alloys with high concentration of alloying elements (e.g., $>0.1 \% \mathrm{Cu},>1.5 \% \mathrm{Mn},>1 \% \mathrm{Ni}$ ), and accumulation of radiationinduced lattice defects, i.e., the so-called matrix features [2, 4]. Both CRPs and MNPs form in medium or high Cu-concentration $(\geqslant 0.1 \% \mathrm{Cu}$ )) alloys, and only MNPs (or Mn/Ni-rich clusters) may form in low (or no) $\mathrm{Cu}$-concentration $(<0.1 \% \mathrm{Cu})$ alloys [5]. In low-alloying steel alloys, only solute clusters or local atmosphere with high solute concentration form. Both precipitates and matrix features can impede dislocation motion [6], causing hardening and embrittlement. Due to the slow kinetics involved, these effects may take several or tens of years to develop, making experimental studies difficult. Alternatively, predictive modeling with validations using the limited experimental data [7] has become appealing.

In RPVs, precipitation takes place at the atomic scale, with the sizes of precipitates in the order of nanometer. Accordingly, most current modeling studies, primarily atomic-kinetic-Monte-Carlo (AKMC) simulations [8-11], have focused on the atomic scale for precipitation kinetics. Assuming homogeneous precipitation in a uniform bcc matrix, good agreement has been demonstrated between AKMC simulations and thermal aging experiments $[12,13]$. However, in reality thermal aging and neutron irradiation take

\footnotetext{
${ }^{*}$ Corresponding author

Email address: yongfeng.zhang@inl.gov (Yongfeng Zhang)
} 
place simultaneously, and solute precipitation and formation of matrix features may affect each other [1416]. Precipitation of alloying elements requires diffusion of solute atoms, usually via exchange mechanism with vacancies or the mixed-dumbbell mechanism $[17,18]$. The precipitation kinetics can be drastically enhanced by radiation-induced point defects, i.e., vacancies and self-interstitial-atoms (SIAs) [19]. Moreover, heterogeneous precipitation may occur at extended defects including pre-existing ones and those produced by neutron irradiation, as evidenced by experimental observations [14]. On the other hand, the presence of alloying elements may change the mobility of vacancies and interstitials, and thus their kinetic evolution. For instance, $\mathrm{Ni}$ and $\mathrm{Mn}$ have been shown to suppress the growth of interstitial loops in bcc Fe-based alloys, resulting in smaller average loop size and narrower size distribution [15]. Therefore, it is critical to fully understand the interaction between solute elements and radiation-induced lattice defects. Recently, simulations of concurrent thermal aging and neutron-irradiation have also been realized to elucidate the effect of radiation conditions $[18,20]$. However, due to the rigid-lattice framework adopted in the AKMC models, the exact representation of extended lattice defects such as prismatic loops was not feasible, and the interaction between precipitates and lattice defects was limited to small defect clusters.

As a starting point to understand $\mathrm{Cu}$ precipitation in realistic RPV alloys, molecular dynamics (MD) and Metropolis-Monte-Carlo (MMC) simulations based on an empirical interatomic potential were utilized to investigate $\mathrm{Cu}$ segregation/precipitation at lattice defects. MD Simulations are used to calculate the interaction between $\mathrm{Cu}$ and various lattice defects, and $\mathrm{MMC}$ simulations with a FeCu1\% model alloy are performed to demonstrate the thermodynamic effect of such interaction. Further consideration of the effect of solute content will be conducted in the future by using more realistic alloying concentrations. Previously, the interaction between $\mathrm{Cu}$ and vacancy clusters [21, 22] or SIA clusters [23] in bcc Fe has been studied using atomistic simulations. In this work, the interaction was extended to extended lattice defects including dislocations, voids and prismatic loops. The purposes of these calculations are to elucidate the nature of interaction between $\mathrm{Cu}$ and these lattice defects, and to compare the effects of various types of defects. We used MD simulations to calculate the segregation energies of $\mathrm{Cu}$ at these defects. MMC simulations were performed to study the possible segregation/precipitation of $\mathrm{Cu}$ at extended lattice defects. The corresponding segregation strengths of these defects were evaluated based on the binding energies. The results, while elucidating the interaction nature between the alloying elements and lattice defects, are also useful for the development of AKMC models with extended lattice defects such as dislocations and loops.

\section{Methods}

Both MD and MMC simulations require an empirical potential to describe the interatomic interaction. Here we use the embedded-atom-method (EAM) potential developed by Pasianot and Malerba (referred to as the Pasianot potential) [24] for Fe-Cu system. In this potential, the Fe-Fe interaction is taken from Mendelev et al. (potential 2) [25] and Cu-Cu interaction from Mishin et al. [26]. The $\mathrm{Fe}-\mathrm{Cu}$ cross interaction is fitted to the experimental Fe-Cu phase diagram and density-functional-theory (DFT) calculations on the point defect-solute interaction and vacancy migration barriers [24]. Developed primarily for the microstructure evolution of $\mathrm{FeCu}$ alloy under radiation, this potential is suitable for studying the interaction between $\mathrm{Cu}$ and lattice defects in bcc Fe.

MD simulations were used to calculate the segregation energy of $\mathrm{Cu}$ near various types of lattice defects which were embedded in a bcc Fe matrix. The defects considered here include vacancies, self-interstitialatoms (SIAs), voids, dislocations and prismatic loops of either vacancy or interstitial type. Among these defects, dislocations are usually pre-existing before irradiation. Others can be produced by neutron irradiation in RPV steels [1]. In the simulations, a vacancy was created by removing a Fe atom, and a SIA by inserting a $\langle 110\rangle$ dumbbell symmetrically at a bcc lattice site, which is the most stable SIA configuration in bcc Fe as shown by previous DFT calculations [27]. Dislocations of either edge or screw type were created by applying the corresponding displacement fields given by the linear-elasticity theory [28] so that a dislocation will be introduced into the system. A void was created by cutting out a spherical region with a radius $r$ in the bcc matrix. For SIA loops, parallel $\langle 111\rangle$ or $\langle 100\rangle$ dumbbells were introduced for the $\langle 111\rangle / 2$ and the $\langle 100\rangle$ loops, respectively. Vacancy loops were constructed by removing one or two layers of atoms with subsequent shifting of nearby atoms according to the Burgers vectors. All prismatic loops were circular in 
shape and pure edge in character. In all simulations, cubic simulation cells were used. Periodic boundary condition was applied along all three directions except for simulations involving dislocations, where periodicity was applied only along the Burgers and line vectors directions (note that they are the same for screw dislocations). The sizes of the simulation cells varied with the types and sizes of the defects being considered, and they all have been shown not to impact the predicted behavior. After being constructed, the simulation cells were quenched dynamically at $0 \mathrm{~K}$ to find the local minimum. The quenching was done by setting the velocity $v_{i}$ of an atom $i$ to zero if $f_{i} \cdot v_{i} \leq 0 ; f_{i}$ is the force acting on atom $i$. Compared with the molecular static (MC) approach which uses minimization to find the local minimum, dynamic quenching allows for the system to overcome some infinitesimal barriers so that a more stable state, if any, can be reached. After quenching, the simulation cells were further relaxed at $300 \mathrm{~K}$ until the potential energy converged to relax the defect configurations.

To get the segregation energy, the total system energy with one $\mathrm{Cu}$ substitution was calculated at $0 \mathrm{~K}$ by quenching. For each defect being considered, a three dimensional segregation energy map was obtained by putting the $\mathrm{Cu}$ atom at each atomic site around the defect. The segregation energy of $\mathrm{Cu} E^{s e g}\left(r_{i}\right)$ at a position $r_{i}$ is defined as $E^{s e g}\left(r_{i}\right)=E\left(r_{i}\right)-E(r=\infty)$, i.e., the excess energy required to bring a substitutional $\mathrm{Cu}$ atom from infinity to the position $r_{i}$. Here $E\left(r_{i}\right)(E(r=\infty))$ is the total system energy with the $\mathrm{Cu}$ atom at position $r_{i}$ (infinity). By this definition, a preferential segregation site is indicated by a negative segregation energy. The binding energy, defined as the negative segregation energy with the largest magnitude, can also be obtained from the results.

The possible segregation/precipitation morphologies of $\mathrm{Cu}$ around the extended defects were explored using MMC simulations. The simulation cells used were similar to those used for segregation energy calculations. Here, $\mathrm{Cu}$ atoms of $1 \%$ atomic percent were randomly introduced into the system as substitutions. MMC simulations were then performed for 5.0 million (5.0 M) MMC steps. During each MMC step, two independent moves, a translation and a swap, were attempted. For the translation, a randomly chosen atom was displaced by a random vector with a length no bigger than $0.1 \stackrel{\AA}{A}$. In the swap, the position of a randomly chosen $\mathrm{Cu}$ atom was swapped with that of a randomly chosen $\mathrm{Fe}$ atom. The attempted moves were accepted if $\varepsilon_{0} \leqslant \exp \left(-\left(E_{f}-E_{i}\right) / K_{B} T\right)$ at finite temperature T. Here $E_{i}$ and $E_{f}$ are the system energies before and after the attempted moves; $\varepsilon_{0}$ is a random number ranging from 0 to $1 ; K_{B}$ is the Boltzmann constant; $\mathrm{T}$ is temperature in unit of Kelvin. The MMC simulations were done at $0 \mathrm{~K}$ if not stated otherwise. At $0 \mathrm{~K}$, the attempt move will be accepted if $E_{f} \leq E_{i}\left(\exp \left(-\left(E_{f}-E_{i}\right) / K_{B} T\right)=1\right)$, and rejected otherwise $\left(\exp \left(-\left(E_{f}-E_{i}\right) / K_{B} T\right)=0\right)$. The swap move can be regarded as an artificial long-range diffusion of $\mathrm{Cu}$ atoms for possible segregation/precipitation, driven by the total system energy. The translation resembles short range diffusion for possible local relaxation. A similar MMC scheme has been used in previous studies for $\mathrm{Cr}$ segregation at grain boundaries and dislocations in bcc Fe [29, 30]. During the MMC simulations, the system pressure was kept zero by adjusting the box dimensions. Both MD and MMC simulations were performed using the LAMMPS simulation package [31] developed at Sandia National Lab. Pictures for atomic configurations were generated using the software AtomEye [32].

\section{Results and discussion}

\subsection{Segregation energy}

\subsubsection{Cu-point defects interaction}

In Fig. 1, the interaction energy between a $\mathrm{Cu}$ atom and a vacancy (V), a SIA, or another $\mathrm{Cu}$ atom are plotted as functions of distance between the pairs of defects. The interaction is important only within the first and second nearest-neighbor $(\mathrm{NN})$ distance. For $\mathrm{Cu}-\mathrm{Cu}$ and $\mathrm{Cu}-\mathrm{V}$, the interaction is attractive and very similar to each other, owing to the high heat of mixing of $\mathrm{Cu}$ in bcc Fe, about $0.55 \mathrm{eV}$ as given by DFT calculations [17]. Such attractive interaction suggests a strong precipitation tendency of $\mathrm{Cu}$. Experimentally, precipitation of $\mathrm{Cu}$ in bcc Fe has been widely observed during thermal aging $[12,13,33]$. In medium to high $\mathrm{Cu}$-concentration $\mathrm{RPV}$ steels, $\mathrm{Cu}$ was observed to precipitate readily and served as precursors for MNPs [5]. In contrast, the interaction between $\mathrm{Cu}$ and SIA is repulsive, consistent with the fact that $\mathrm{Cu}$ is an oversized solute in bcc Fe. The highest repulsion is obtained when a mixed dumbbell forms with $\mathrm{Cu}$ 


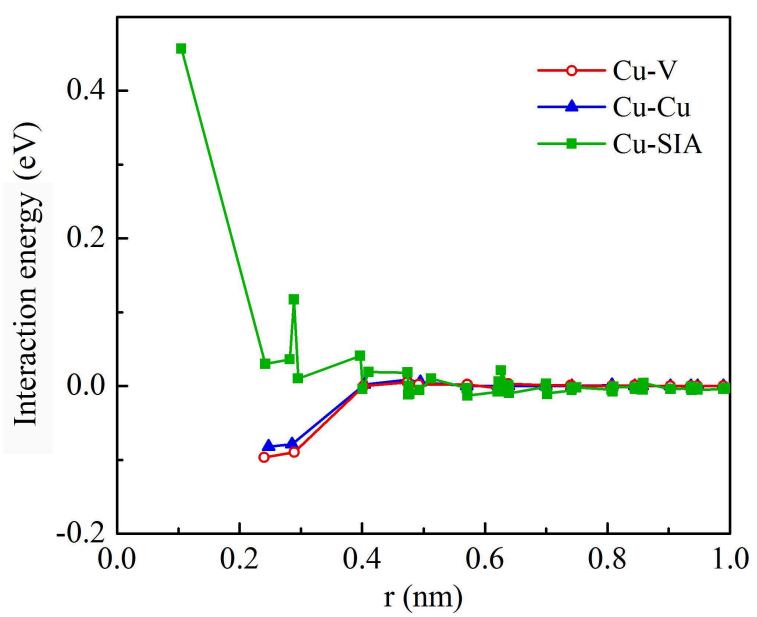

Figure 1: (Color online) Interaction energies between $\mathrm{Cu}$ and point defects or another $\mathrm{Cu}$ atom, as functions of distance. The $\mathrm{Cu}-\mathrm{Cu}$ and $\mathrm{Cu}-\mathrm{V}$ interaction is attractive, while the $\mathrm{Cu}-\mathrm{SIA}$ interaction is repulsive.

occupying one of the dumbbell sites. Thus, the possibility for $\mathrm{Cu}$ to diffuse via mixed-dumbbell mechanism is very low. However, SIAs may still affect $\mathrm{Cu}$ diffusion and precipitation by recombining with vacancies and thus reducing the available vacancy concentration. On the other hand, the presence of $\mathrm{Cu}$ may affect the diffusion of SIA [34] or SIA clusters [23], by altering the configuration-dependent formation energies in order to reduce the repulsive interaction.

To compare with previous MD studies [24, 35, 36] and DFT calculations [17, 37-42], the binding energies for $\mathrm{Cu}-\mathrm{V}, \mathrm{Cu}-\mathrm{Cu}$ and $\mathrm{Cu}-\mathrm{SIA}$ defect pairs are listed in Table 1. Our results are almost identical to those given by Pasianot et al. [24] since they are produced by the same potential. The Pasianot potential more accurately predicts the $\mathrm{Cu}-\mathrm{SIA}$ interaction, while predicting the $\mathrm{Cu}-\mathrm{Cu}$ and $\mathrm{Cu}-\mathrm{V}$ interaction with about the same accuracy, in comparison with previous empirical potentials $[35,36]$ by taking the DFT results as references.

Table 1: $\mathrm{Cu}-\mathrm{V}, \mathrm{Cu}-\mathrm{SIA}$ and $\mathrm{Cu}-\mathrm{Cu}$ binding energies $(\mathrm{eV})$. Positve binding energies mean attraction and negative mean repulsion.

\begin{tabular}{|c|c|c|c|c|c|c|c|c|c|c|c|}
\hline \multirow{2}{*}{$\begin{array}{c}\text { Interaction } \\
(\mathrm{eV})\end{array}$} & \multirow{2}{*}{ Present } & \multicolumn{3}{|c|}{ MD } & \multicolumn{7}{|c|}{$\overline{\mathrm{DFT}}$} \\
\hline & & $\mathrm{Pa}[24]$ & $\mathrm{Lu}[35]$ & $\mathrm{Ac}[36]$ & Do[37] & $\mathrm{Vi}[43]$ & $\mathrm{Be}[38]$ & So $[39]$ & Go $[40]$ & $\mathrm{Ol}[41]$ & $\mathrm{Oh}[42]$ \\
\hline $\mathrm{Cu}-\mathrm{V}(1 \mathrm{NN})$ & 0.10 & 0.10 & 0.19 & 0.09 & 0.17 & & 0.17 & 0.17 & 0.26 & 0.27 & 0.24 \\
\hline $\mathrm{Cu}-\mathrm{V}(2 \mathrm{NN})$ & 0.09 & 0.09 & -0.03 & 0.04 & 0.19 & & 0.28 & 0.18 & 0.21 & 0.16 & \\
\hline $\mathrm{Cu}-\mathrm{SIA}(1 \mathrm{NN})$ & -0.46 & -0.45 & 0.07 & -0.08 & -0.43 & -0.46 & & & & & \\
\hline $\mathrm{Cu}-\mathrm{SIA}(2 \mathrm{NN})$ & -0.03 & -0.03 & 0.09 & 0.02 & 0.10 & $0.07 /-0.01$ & & & & & \\
\hline $\mathrm{Cu}-\mathrm{Cu}(1 \mathrm{NN})$ & 0.08 & 0.08 & 0.19 & 0.08 & 0.14 & & 0.14 & 0.15 & & & \\
\hline $\mathrm{Cu}-\mathrm{Cu}(2 \mathrm{NN})$ & 0.08 & 0.08 & -0.02 & 0.04 & 0.03 & & 0.03 & 0.03 & & & \\
\hline
\end{tabular}

\subsubsection{Cu-dislocation interaction}

Unlike point defects which only have short range interaction with $\mathrm{Cu}$, dislocations may have long range interaction with $\mathrm{Cu}$ through their associated stress fields. For an edge dislocation, depending on the location, a $\mathrm{Cu}$ atom can be either attracted or repelled from the dislocation core, as shown in Fig. 2(a). The attraction takes place at the tensile stress region and the repulsion at the compressive region. The interaction strength decreases with increasing distance from the core, with non-zero segregation energies even at long range. In contrast, a screw dislocation attracts $\mathrm{Cu}$ only at short range, and the interaction is weaker than that displayed by an edge dislocation. The existence of attractive regions indicates that both edge and screw dislocation can be preferential precipitation sites for $\mathrm{Cu}$.

Ideally, solute with a size mismatch $\Delta V$, i.e., volumetric change induced by replacing one matrix atom with a solute, interacts elastically with a stress field. The corresponding contribution to segregation energy 


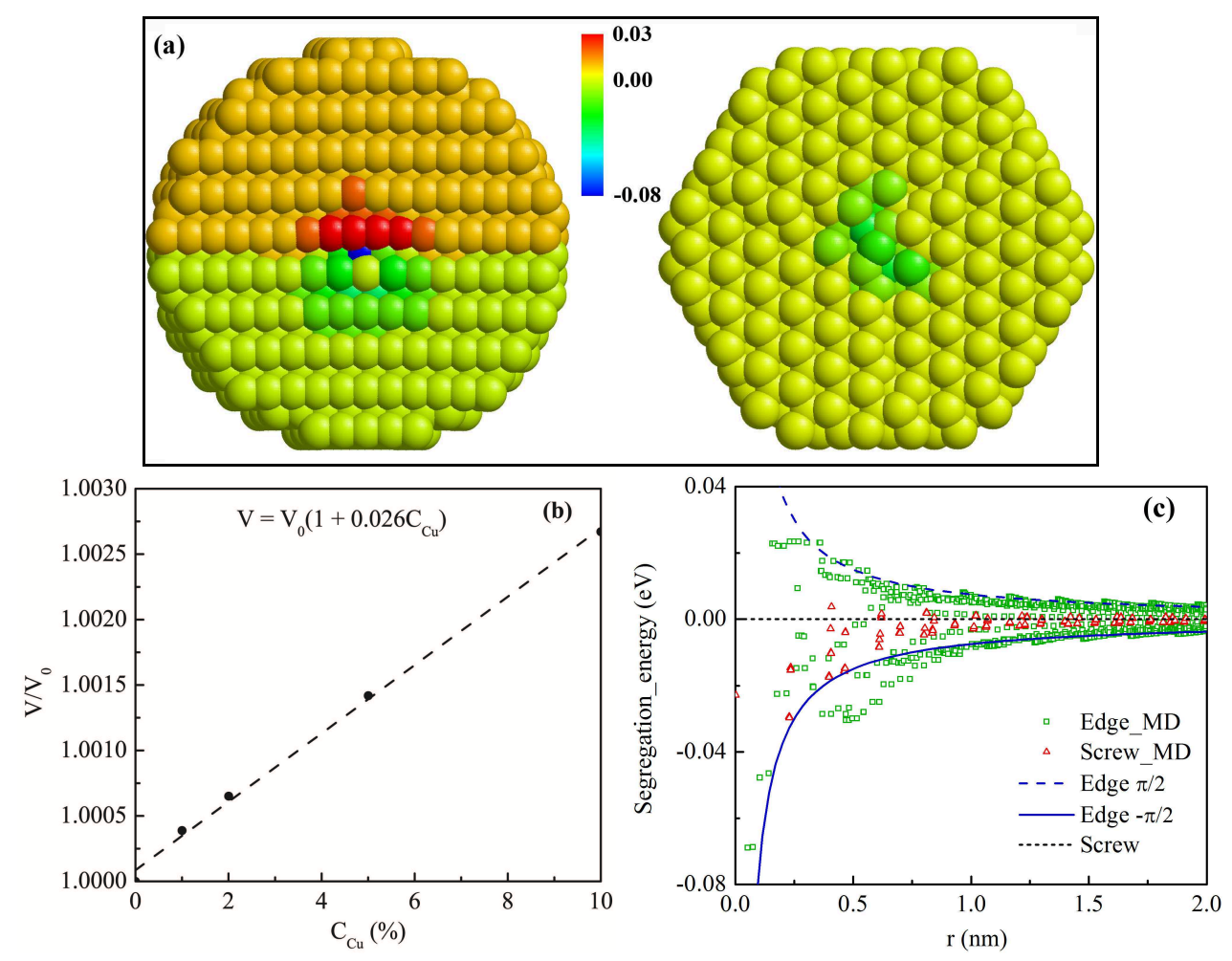

Figure 2: (Color online) (a) Cu segregation energy (in eV) maps around the cores of an edge (left) and a screw dislocation (right), (b) atomic volume of bcc Fe as a function of $\mathrm{Cu}$ concentration, and (c) Cu segregation energies as functions of distances from the dislocation cores. The symbols represent MD results and the curves represent the linear-elasticity predictions. The segregation energies from MD calculations agree well with the predictions based on elastic interaction.

is given by $\Delta E=-P \Delta V$; here $P$ is the hydrostatic pressure [28]. Oversized solutes are attracted by tension and undersized ones by compression. Therefore, the elastic interaction can be estimated if the size mismatch and hydrostatic pressure are known. To determine the size mismatch of $\mathrm{Cu}$ in bcc Fe, $\mathrm{MD}$ simulations were performed to calculate the atomic volume $V$ of bcc Fe with various $\mathrm{Cu}$ concentrations $C_{C u}$. $\mathrm{Cu}$ atoms were randomly inserted into substitutional sites. As shown in Fig. 2(b), $V$ increases proportionally to $C_{C u}$. Linear fitting gives $V\left(C_{C u}\right)=V_{0}+C_{C u} \Delta V=V_{0}\left(1+0.026 C_{C u}\right)$; here $V_{0}$ is the atomic volume of bcc Fe and it is $11.64 \AA^{3}$ given by the Mendelev potential [25]. Correspondingly, the size mismatch is determined as $\Delta V=0.026 V_{0}$ for $\mathrm{Cu}$, close to the value of $0.021 V_{0}$ given by another interatomic potential [34]. The hydrostatic pressure of an edge dislocation with anisotropic elastic moduli can be determined using the linear-elasticity theory with the Stroh formalism [44]. In the calculation we used infinite long straight dislocations with the Burgers vectors $b=\frac{1}{2}\langle 111\rangle$. The elastic moduli used for bcc Fe are $\mathcal{C}_{11}=243.4 \mathrm{GPa}$, $\mathcal{C}_{12}=145 \mathrm{GPa}$, and $\mathcal{C}_{44}=116 \mathrm{GPa}$, as given by the Mendelev potential [25]. The hydrostatic pressure induced by a screw dislocation is zero according to linear-elasticity theory [28].

In Fig. 2(c), the segregation energies of $\mathrm{Cu}$ near an edge and a screw dislocation are plotted as functions of distances from the dislocation cores. In both MD and theoretic calculations, a coordination system was used so that the maximum tension of an edge dislocation is along the $\theta=-\pi / 2$ direction and maximum compression along the $\theta=\pi / 2$ direction, i.e., the extra atomic plane. For the edge dislocation, most MD data are enclosed by the two theoretical curves representing the maximum attraction $(\theta=-\pi / 2)$ and the maximum repulsion $(\theta=\pi / 2)$. Some deviation appears when the distance is less than $1 \mathrm{~nm}$, or a few $a_{0}(2.85 \AA)$, the lattice constant of bcc Fe. Within this range the interaction becomes asymmetric with slightly stronger attraction than repulsion. For the screw dislocation, linear-elasticity theory predicts zero interaction. In contrast, $\mathrm{MD}$ simulations indicate that $\mathrm{Cu}$ atoms are attracted at the core region within a 
couple of $a_{0}$.

The results shown in Fig. 2 suggest that the interaction between $\mathrm{Cu}$ and a dislocation can be divided into two parts: elastic interaction via the associated stress field and the core effect which presents only within a few $a_{0}$, or a few Burgers vector $b$. For the edge dislocation, the interaction is dominated by the elastic effect with good agreements between MD and theoretic calculations even at locations near the core. Such elasticity-dominated interaction with edge dislocations has also been observed for C in bcc Fe [45], and $\mathrm{H}$ in fcc $\mathrm{Ni}$ [46]. For a screw dislocation, the interaction is dominated by the core effect owing to the negligible hydrostatic pressure at long range. The core effect is generally attractive for $\mathrm{Cu}$. For the edge dislocation, incorporating the core effect shifts the interaction with $\mathrm{Cu}$ towards the attraction side, giving an asymmetric segregation energy profile in contrast to the symmetric curves predicted by linear elastic theory. We note that the interaction between $\mathrm{Cu}$ and dislocations obtained from MD simulations is dependent on the choice of interatomic potential. The Mendelev potential has been shown able to reproduce the screw dislocation core configurations in reference to DFT calculations [47]. Its performance on the cores of edge dislocations is unclear. This may affect the part of core effect in the interaction. The Mendelev potential has been fitted using the elastic properties of bcc Fe and thus the elastic stress field is in good agreement with linear elasticity theory prediction. Some uncertainty in the elastic interaction part may come from the size-mismatch. According to a recent DFT calculations, the volumetric mismatch between bcc $\mathrm{Cu}$ and bcc Fe is about $5.4 \%$ (derived from $1.8 \%$ lattice mismatch) [48], larger than the value of $2.6 \%$ given above. Therefore, an even stronger elastic interaction between $\mathrm{Cu}$ and stress fields may exist than that predicted in this work.
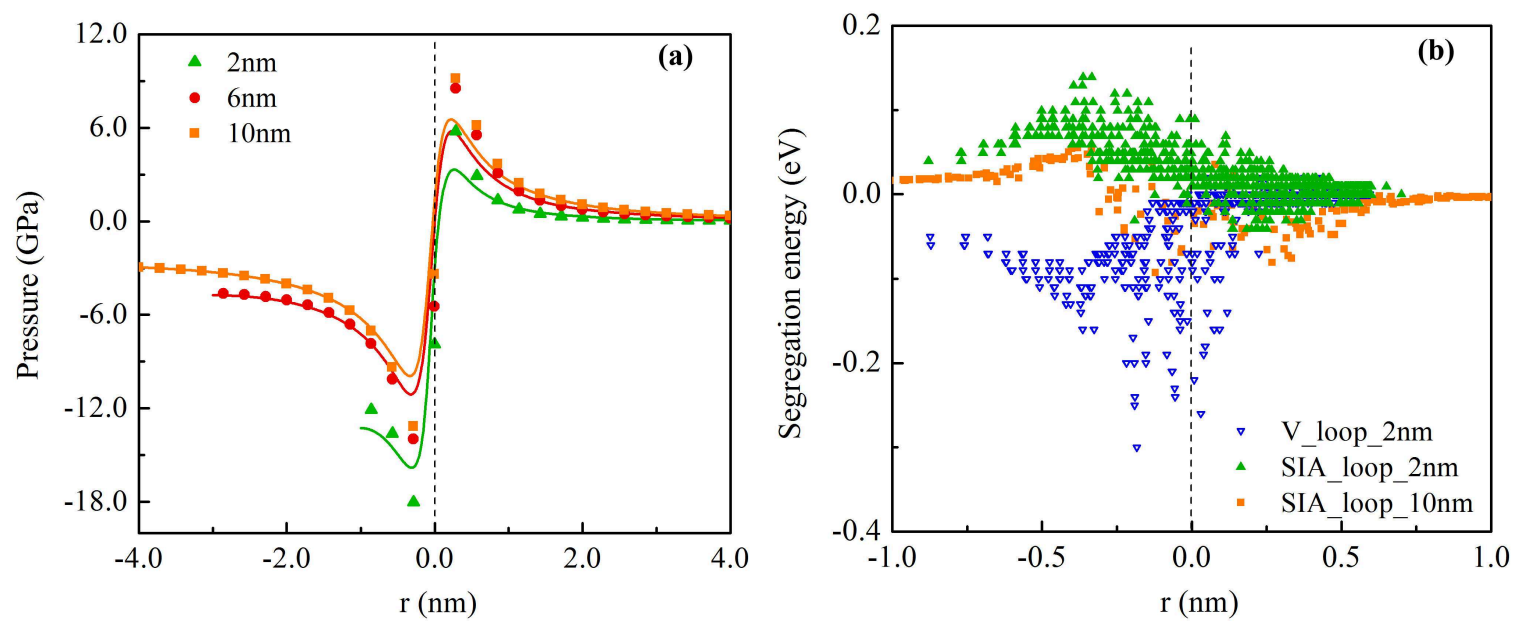

Figure 3: (Color online) (a) Hydrostatic pressure and (b) $\mathrm{Cu}$ segregation energies as functions of distances from the loop edges (denoted by vertical dash lines). Positive pressure means tension and negtive pressure means compression. In (a) Symbols represent MD results and the curves are from Stroh formalism. The stress fields of loops given by MD calculations can be well reproduced by the linear-elasticity theory.

\subsubsection{Cu-loop interaction}

Prismatic loops have been widely observed in neutron and ion irradiated Fe-based alloys [1, 49, 50]. Most loops are characterized as SIA type in nature, while vacancy loops may also form near surfaces [49]. The loops are found to have either a $\frac{1}{2}\langle 111\rangle$ or a $\langle 100\rangle$ Burgers vector [50]. In this work, both $\frac{1}{2}\langle 111\rangle$ or $\langle 100\rangle$ loops have been considered. In the following, only the results for $\langle 100\rangle$ loops will be presented. Similar but weaker interaction with $\mathrm{Cu}$ were obtained for $\frac{1}{2}\langle 111\rangle$ loops owing to the smaller Burgers vector and thus lower stress field.

Analogous to the edge dislocation (note that the prismatic loops are pure edge in character), the interaction between $\mathrm{Cu}$ and prismatic loops are also dominated by elastic effect via the associated stress fields. Unlike a straight edge dislocation, for which the stresses along the $-\pi / 2$ and $\pi / 2$ directions are symmetric 
with opposite signs, the stresses are not symmetric across the loop edge (positive and negative directions along $r$ ) due to stress overlapping. This asymmetry is clear in Fig. 3(a) where the hydrostatic pressures calculated using MD simulations are plotted for SIA loops of various sizes. In the MD calculations, atomic stress was averaged over atoms in a slab given by $|z| \leqslant b$; here $z$ is the distance from the loop residing plane. The pressure given by MD simulations compares well with that predicted by the linear-elasticity theory using the Stroh formalism [44], except for regions near the core. In the Stroh formalism, again we used the elastic moduli given by the Mendelev potential. A cutoff of $1.0 b$ is used for the loop core in order to compare with the average atomic stress calculated in the same range.

Across the loop edges (with the core corresponding to $r=0$ ), the pressure changes sign and so does the $\mathrm{Cu}$ segregation energy, as shown in Fig. 3(b). Here positive pressure means tension and negative means compression. For SIA loops (SIA loop), the stress is compressive inside $(r<0)$ and tensile outside $(r>0)$. Correspondingly, the interaction with $\mathrm{Cu}$ is repulsive inside and attractive outside. For vacancy loops (V_loop), the signs of the pressure and the interaction with $\mathrm{Cu}$ are the opposites to those for SIA loops. Due to stress field overlapping, at the same distance from the edge the pressure inside the loop is higher in magnitude than that outside. For SIA (vacancy) loops, this means higher compression (tension) inside than tension (compression) outside, as shown in Fig. 3(a). Thus, SIA (vacancy) loops always exhibit stronger repulsion (attraction) to $\mathrm{Cu}$ inside than attraction (repulsion) outside. Theoretically, the stress filed of a vacancy loop is symmetrical to that of a SIA loop of the same size and shape. Therefore, in magnitude, the attraction to $\mathrm{Cu}$ inside a vacancy loop equals the repulsion inside a SIA loop of the same size, and both are higher than the attraction outside the SIA loop. In other words, a vacancy loop is thermodynamically a more preferred segregation site to $\mathrm{Cu}$ than a SIA loop of the same size, as indicated by the segregation energy profiles shown in Fig. 3(b). We note that the segregation energy profiles of a vacancy loop and a SIA loop are usually not exactly symmetric to each other because of the core effect, which, as discussed earlier, shifts the interaction with $\mathrm{Cu}$ towards the attraction side.

The stress field overlapping of loops depends on their sizes. As the size increases, the overlapping effect diminishes. For a SIA loop, the maximum compression inside decreases and the maximum tension outside increases, as shown in Fig. 3(a). Accordingly, the maximum attraction to $\mathrm{Cu}$ will increase, indicating higher segregation strength with larger loop size. Vacancy loops behave in the opposite way as SIA loops do. At infinite size, the pressure becomes symmetric and approaches that of a straight edge dislocation for both vacancy and SIA loops.

\subsubsection{Cu-void interaction}

Due to the high heat of mixing of $\mathrm{Cu}$ in bcc Fe, void surfaces, or in general free surfaces, are expected to be preferential precipitation sites. Indeed, $\mathrm{MD}$ calculations show that the binding energies of $\mathrm{Cu}$ at the surface of a $2 \mathrm{~nm}$ (diameter) void can be as high as $0.67 \mathrm{eV} . \mathrm{Cu}$ atoms are attracted by the void surface in a range of about one $\mathrm{a}_{0}$, followed by a slightly repulsive zone with positive segregation energies, as shown in Fig. 4. This result is in agreement with previous studies which showed that strong affinity exists between $\mathrm{Cu}$ and vacancy type defects $[21,22]$.

Table 2: $\mathrm{Cu}$ binding energies at various lattice defects. Positive binding energies mean attraction and negative mean repulsion.

\begin{tabular}{ccccccccc}
\hline \hline Defect & vacancy & SIA & Edge & Screw & $\begin{array}{c}\text { V_loop } \\
(2 \mathrm{~nm})\end{array}$ & $\begin{array}{c}\text { SIA_loop } \\
(2 \mathrm{~nm})\end{array}$ & $\begin{array}{c}\text { SIA_loop } \\
(10 \mathrm{~nm})\end{array}$ & $\begin{array}{c}\text { Void } \\
(2 \mathrm{~nm})\end{array}$ \\
\hline Binding energy $(\mathrm{eV})$ & 0.10 & -0.46 & 0.08 & 0.03 & 0.30 & 0.04 & 0.09 & 0.67 \\
\hline
\end{tabular}

In RPV steels, all the above considered defects may co-exist and they compete with each other as potential precipitation sites. Therefore it is of interest to compare their relative segregation strengths. For this purpose, the segregation energies of $\mathrm{Cu}$ around these defects are plotted in Fig. 4, and the corresponding binding energy (the negative of segregation energy, maximum in magnitude) are summarized in Table 2. Due to the high heat of mixing, vacancy type defects (vacancy and void) in general display higher binding energies of $\mathrm{Cu}$ than SIA type defects. Such interaction is usually in short range. Long range interaction with $\mathrm{Cu}$ presents for defects with long-range stress fields, such as edge dislocations and prismatic loops. In 
these cases, the interaction is dominated by elastic effect and can be well described by the linear-elasticity theory. As an oversized solute, $\mathrm{Cu}$ is attracted by tensile stress fields and repelled by compressive ones. The magnitude of elastic interaction is proportional to the hydrostatic pressure, which is size dependent for prismatic loops.

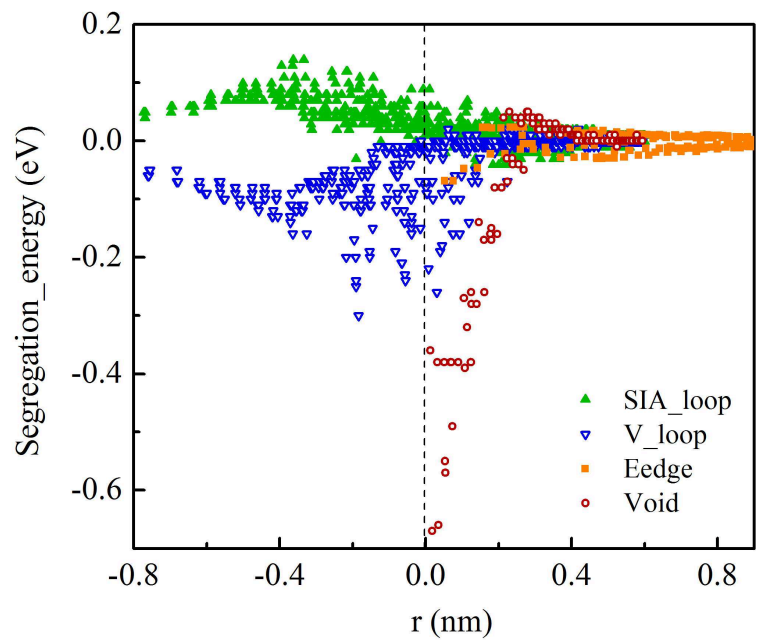

Figure 4: (Color online) $\mathrm{Cu}$ segregation energies as functions of distances for an edge dislocation, prismatic loops $(2 \mathrm{~nm}$ in diameter) and a void. The vertical dash line denotes the positions of the void surface and the cores of loops and dislocations. Vacancy type defects exhibit stronger attraction to $\mathrm{Cu}$ than SIA type ones do.

\subsection{Precipitation morphology}

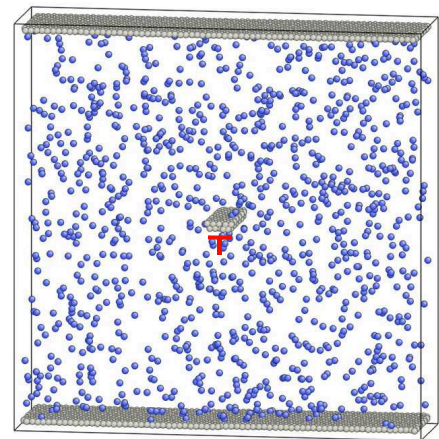

(a)

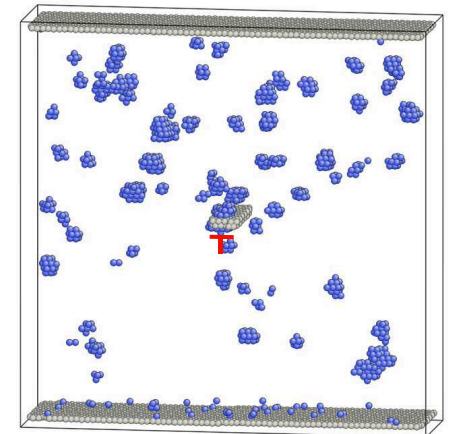

(b)

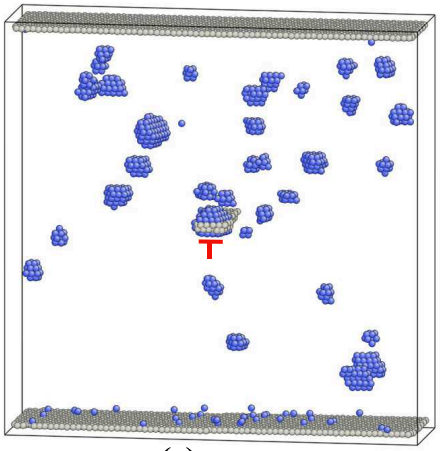

(c)

Figure 5: (Color online) Atomic configurations to demonstrate $\mathrm{Cu}$ precipitation around an edge dislocation at $0 \mathrm{~K}$ after (a) 0 , (b) $2.5 \mathrm{M}$ and (C) 5.0M MMC steps. Cu atoms are show in blue and non-bcc Fe atoms in gray spheres representing the dislocation core and free surfaces. Due to the tensile stress above the dislocation line, many more precipitates form there than below the dislocation line.

The results on segregation energies suggest that all above considered defects may act as heterogeneous nucleation sites for $\mathrm{Cu}$ precipitates. In this section, the possible precipitation morphology at these defects are explored using MMC simulations.

The precipitation results of $\mathrm{Cu}$ near an edge dislocation are illustrated in Fig. 5. The simulation cell was $4.2 \mathrm{~nm}$ along the dislocation line, and about $18.0 \mathrm{~nm}$ along the other two directions. To avoid $\mathrm{Cu}$ segregation at free surfaces (top and bottom), no MMC moves were performed for atoms within $1.0 \mathrm{~nm}$ from surfaces. Randomly distributed initially, most $\mathrm{Cu}$ atoms except those in the fixed surface layers formed 
clusters within 2.5 M MMC steps; Fig. 5(a)-(b). Due to the favorable tensile stress above the dislocation line, clear preferential precipitation can be observed in this region: there were many more $\mathrm{Cu}$ precipitates above than below the dislocation line. Decoration of dislocation line by a $\mathrm{Cu}$ precipitate was also observed. Experimentally, $\mathrm{Cu}$ precipitation at dislocation lines has been detected by atom-probe-tomography (APT) in both an Fe-Cu0.1wt\% model alloy [14] and a RPV steel [51]. Within another 2.5 M MMC steps, some small precipitates disappeared and the relatively large ones kept growing in size by absorbing $\mathrm{Cu}$ atoms dissolved from small ones, showing a ripening behavior [52] (see Fig. 5(b)-(c)). During this period, the total number of $\mathrm{Cu}$ clusters decreases and the average size increases. In a separate simulation with a screw dislocation, no apparent preferential nucleation was observed except at the core, where decoration of dislocation line by a $\mathrm{Cu}$ precipitate was also characterized.



(a)

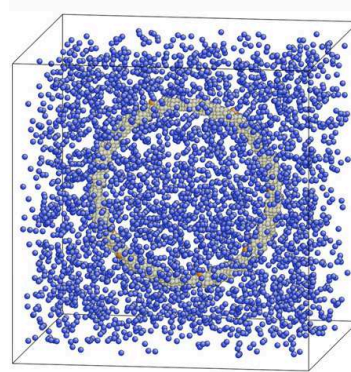

(d)

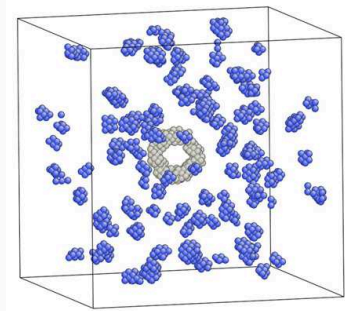

(b)

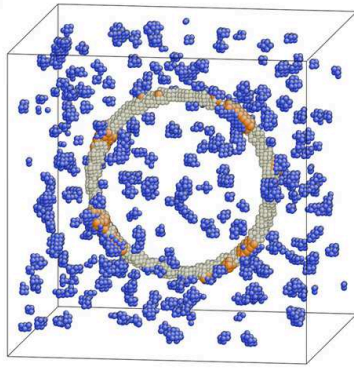

(e)

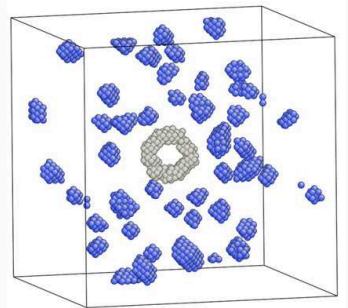

(c)

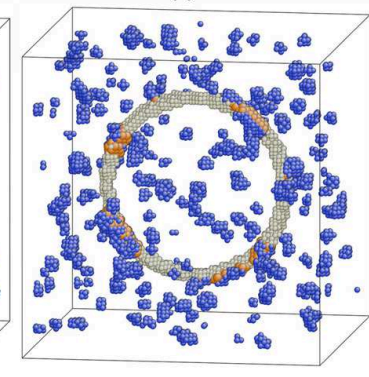

(f)



(g)

Figure 6: (Color online) Atomic configurations to demonstrate $\mathrm{Cu}$ precipitation around a $2 \mathrm{~nm}$ SIA loop at $0 \mathrm{~K}$ after (a) 0 , (b) $2.5 \mathrm{M}$ and (c) $5.0 \mathrm{M}$ MMC steps; and that around a $10 \mathrm{~nm}$ SIA loop at (d) 0 , (e) $2.5 \mathrm{M}$ and (f) $5.0 \mathrm{M} \mathrm{MMC} \mathrm{steps.} \mathrm{bcc} \mathrm{Cu}$ atoms are show in blue; non-bcc $\mathrm{Cu}$ atoms are shown in yellow; and non-bcc Fe atoms are shown in gray spheres to represent the loop. In $(\mathrm{g})$ the local $\mathrm{Cu}$ concentration $\mathrm{C}_{C u}$ is plotted as a function of distance $\mathrm{r}$ from the loop center, with the loop edges denoted by dash lines. Decoration by $\mathrm{Cu}$ precipitates is observed for the $10 \mathrm{~nm}$ SIA loop but not the $2 \mathrm{~nm}$ one.

Figure 6 shows the precipitation of $\mathrm{Cu}$ around two SIA loops, with the sizes being $2 \mathrm{~nm}$ and $10 \mathrm{~nm}$ respectively. The simulation cells were 11.5 by 11.5 by $11.5 \mathrm{~nm}^{3}$ for the $2 \mathrm{~nm}$ loop, and 16.1 by 16.1 by $17.2 \mathrm{~nm}^{3}$ for the $10 \mathrm{~nm}$ loop. For the $2 \mathrm{~nm}$ loop, all precipitates were found to form in the matrix, with no precipitates near the loop edge (Fig. 6(a)-(c)). While for the $10 \mathrm{~nm}$ loop, decoration of the loop edge by $\mathrm{Cu}$ precipitates (shown as non-bcc $\mathrm{Cu}$ ) can be clearly seen in Fig. 6(e) and (f). To further demonstrate this, the radial profile of local $\mathrm{Cu}$ concentration $\mathrm{C}_{C u}$ is plotted in Fig. $6(\mathrm{~g})$. Here $\mathrm{C}_{C u}$ was calculated within a thin slab of $|z|<0.5 \mathrm{~nm}$, with $|z|=0$ for the loop residing plane. $\mathrm{C}_{C u}$ is defined as the ratio of the number of $\mathrm{Cu}$ atoms over the total number of atoms in each shell depicted by $\left|r_{i}\right|<|r|<\left|r_{i}\right|+a_{0}$. For the $10 \mathrm{~nm}$ loop, a clear peak presents just at the outside of the edge, showing strong $\mathrm{Cu}$ precipitation there. Such a peak is absent for the $2 \mathrm{~nm}$ loop. This result is consistent with the segregation energy calculations which showed that the segregation strengths of SIA loops increase with increasing sizes. In literature, copper clusters segregated at a $10 \mathrm{~nm}$ circular object have been detected by APT in a $\mathrm{Fe} 0.1 \mathrm{Cu}$ wt\% alloy irradiated up to 0.2 dpa [15]. The circular objective was suggested to be a decorated prismatic loop, a configuration similar to that shown in Fig. 6(e)-(f). Using similar MMC simulations, the decoration of prismatic loops by Cr has also been observed previously in a Fe-Cr10\% alloy [30].

Strong precipitation tendency was also observed at the surface of a $2 \mathrm{~nm}$ void, as shown in Fig. 7 . The 
simulation cells were 11.5 by 11.5 by $11.5 \mathrm{~nm}^{3}$ in size. The surface of the void was almost all covered by $\mathrm{Cu}$ atoms within 2.5 M MMC steps, forming a core-shell precipitate configuration similar to that reported earlier $[21,22]$. Within another 2.5 M MMC steps, the spherical void developed into a $\{110\}$ faceted precipitate, owing to the low interface energy for the $\{110\}$ plane [35]. Cu atoms at the first layer of the void surface are in non-bcc local structure, as shown in Fig. 7(b) and (c). Ripening type behavior was also observed with small clusters dissolving and larger ones growing.

In summary, the MMC results on $\mathrm{Cu}$ precipitation are consistent with the segregation energy calculations. Preferential precipitation was characterized at locations with negative segregation energies, such as the exterior of SIA loops and the surfaces of voids. Such preferential precipitation was explained by the high heat of mixing and the positive size mismatch of $\mathrm{Cu}$ in bcc Fe.

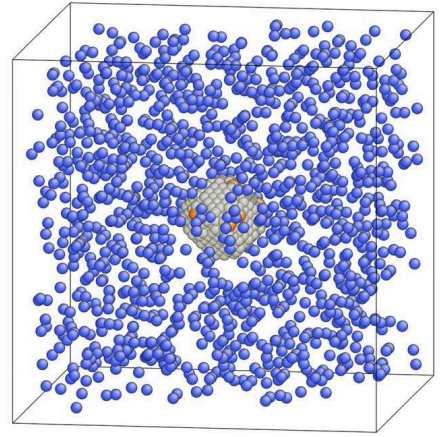

(a)

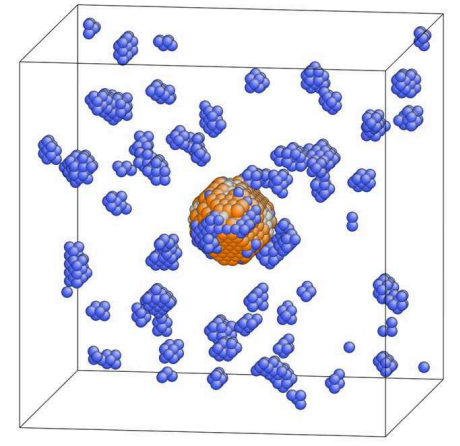

(b)

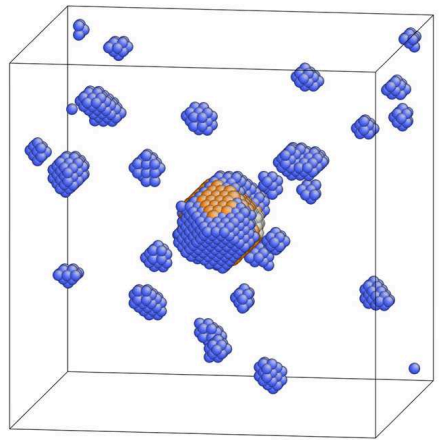

(c)

Figure 7: (Color online) Atomic configurations to demonstrate $\mathrm{Cu}$ precipitation around a $2 \mathrm{~nm}$ void at $0 \mathrm{~K}$ after (a) 0 , (b) $2.5 \mathrm{M}$ and (c) $5.0 \mathrm{M}$ MMC steps. bcc $\mathrm{Cu}$ atoms are show in blue; non-bcc $\mathrm{Cu}$ atoms are shown in yellow; and non-bcc Fe atoms are shown in gray spheres representing void surface. Preferential precipitation of $\mathrm{Cu}$ at the void surface is clear by the formation of faceted precipitates around the void.

\subsection{Discussion}

The current work focuses on thermodynamic aspects on the interaction between $\mathrm{Cu}$ and extended lattice defects in bcc Fe. In both MD calculations and MMC simulations no kinetics of solute diffusion are considered. Consistent with previous experimental and modeling studies, the current results show that the precipitation of alloying elements can be affected by the presence of lattice defects $[14,15]$. Firstly, it is shown that extended defects such as dislocations, voids and loops may induce heterogeneous precipitation of $\mathrm{Cu}$. In previous experiments, it has been observed that the high density of defect clusters produced by ion-irradiation promoted $\mathrm{Cu}$ precipitation in a $\mathrm{FeCu0.1-wt \%} \mathrm{alloy,} \mathrm{and} \mathrm{the} \mathrm{precipitation} \mathrm{kinetics} \mathrm{obtained}$ from experiments can only be explained by considering heterogeneous nucleation at these defect clusters [14]. Such a conclusion was supported by APT reconstruction which showed heterogeneous $\mathrm{Cu}$ precipitation at an edge dislocation (the $\frac{1}{2}\langle 110\rangle$ burger vector reported in the work could possibly be the projection of $\frac{1}{2}\langle 111\rangle$ for bcc Fe) [14]. Heterogeneous $\mathrm{Cu}$ and $\mathrm{Mn}$ and $\mathrm{Ni}$ precipitation at prismatic loops has been suggested by previous experiments $[15,53]$ and recent atomistic modeling which showed that small dislocation loops can act as heterogeneous nucleate sites of precipitates by enhancing their thermodynamic stability [54]. In the present work, decoration of dislocation line was observed in MMC simulations for edge dislocation (Fig. 5)), vacancy loops and SIA loops (Fig. 6). On the other hand, the formation of matrix features is also affected by the presence of alloying elements. For instance, the average loop size measured by transmission-electronmicroscopy analysis decreases with increasing concentration of alloying elements [15]. It is well known that $\langle 111\rangle$ SIA loops are highly mobile along their Burgers vector directions. Decoration by alloying elements and precipitates may reduce the mobility of loops, and thus reduce their chances to grow by meeting other loops or individual point defects.

The interaction between $\mathrm{Cu}$ and matrix features can be understood by the high heat of mixing and the positive size mismatch of $\mathrm{Cu}$ in bcc Fe. The former is responsible for the low solubility of $\mathrm{Cu}$, and it 
leads to strong attraction between $\mathrm{Cu}$ and vacancy type defects or another solute atom. This interaction is usually in short range. Moreover, as an oversized solute, $\mathrm{Cu}$ is found to interact elastically with stress fields associated with dislocations and loops. This interaction can be theoretically formulated without considering the exact defect configurations. This conclusion is very important for AKMC simulations where a rigid lattice framework is used and explicit consideration of extended defects such as loops is not feasible. However, the effect of dislocations and loops on $\mathrm{Cu}$ precipitation may still be considered by incorporating the associated stress field, as has been done in the Fe-C system in a previous work [45].

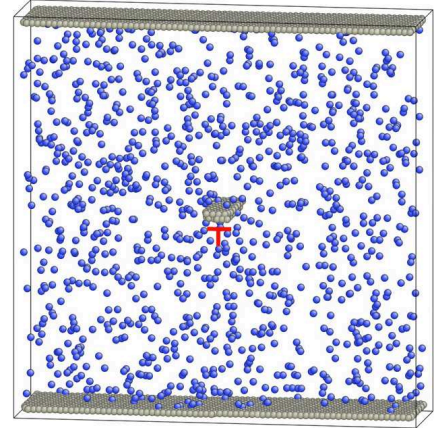

(a)

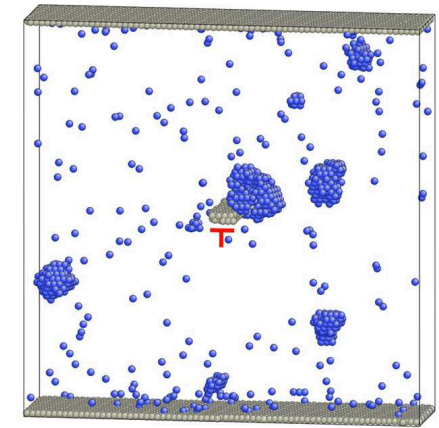

(b)



(c)

Figure 8: (Color online) Atomic configurations to demonstrate $\mathrm{Cu}$ precipitation around an edge dislocation at $573 \mathrm{~K}\left(300^{\circ} \mathrm{C}\right.$ ) after (a) 0, (b) 2.5M and (C) 5.0M MMC steps. Cu atoms are show in blue and non-bcc Fe atoms in gray spheres representing the dislocation core and free surfaces. Higher $\mathrm{Cu}$ solubility than that at $0 \mathrm{~K}$ can be seen by the higher density of isolated $\mathrm{Cu}$ atoms. Decoration of dislocation line by $\mathrm{Cu}$ precipitates was clear in (b) and (c) at this temperature.

Given the relatively weak interaction between $\mathrm{Cu}$ and dislocations and SIA type defects, one may be curious how they will affect $\mathrm{Cu}$ precipitation at the operation temperature of RPVs, which is about $300^{\circ} \mathrm{C}$. To elucidate this, an MMC simulation at $300^{\circ} \mathrm{C}$ was performed using the same simulation cell shown in Fig. 5. As shown in Fig. 8, increasing in temperature increases $\mathrm{Cu}$ solubility in bcc Fe, as indicated by the higher density of isolated $\mathrm{Cu}$ atoms than that at $0 \mathrm{~K}$. Moreover, at $300^{\circ} \mathrm{C}$, the thermal energy becomes comparable to or even higher than the elastic interaction energy between $\mathrm{Cu}$ and an edge dislocation. As a result, the preferential $\mathrm{Cu}$ precipitation induced by elastic interaction with the tensile stress field above the edge dislocation is reduced, similar to the situation in Fe-Cr alloys studied by a similar MMC approach [30]. However, heterogeneous $\mathrm{Cu}$ precipitation at dislocation core was still characterized. This result suggests that due to the small volumetric mismatch between bcc $\mathrm{Cu}$ and $\mathrm{Fe}$, the elastic interaction itself may not be sufficient to establish preferential $\mathrm{Cu}$ precipitation at RPV operation temperatures. However, with the core effect included, heterogeneous precipitation can still occur at dislocations and loops, in agreement with previous experiments $[14,51]$.

\section{Conclusions}

Using $\mathrm{MD}$ and $\mathrm{MMC}$ simulations, in this work the $\mathrm{Cu}$ segregation energies in bcc Fe at extended lattice defects, including dislocations, prismatic loops and voids, are calculated. The results suggest that $\mathrm{Cu}$ has a strong precipitation tendency at vacancy type defects due to the high heat of mixing. This interaction is in short range, usually within a couple of lattice constant. In addition, Cu interact elastically with stress fields. This interaction is in long range and it can be well described by the linear-elasticity theory, allowing for the consideration of edge dislocations and prismatic loops, for instance, in AKMC simulations, without representing their exact atomic configurations. In accordance to the segregation energy calculations, preference $\mathrm{Cu}$ precipitation at these extended lattice defects are observed during MMC simulations. The present results can be used to explain the previous APT experiments on $\mathrm{Cu}$ precipitation in some model alloys and RPV steels. 


\section{ACKNOWLEDGEMENT}

The authors gratefully acknowledge the support of the DOE Light Water Reactor Sustainability (LWRS) Program. This manuscript has been authored by Battelle Energy Alliance, LLC under Contract No. DEAC07-05ID14517 with the U.S. Department of Energy. The United States Government retains and the publisher, by accepting the article for publication, acknowledges that the United States Government retains a nonexclusive, paid-up, irrevocable, world-wide license to publish or reproduce the published form of this manuscript, or allow others to do so, for United States Government purposes.

\section{References}

[1] W. J. Phythian and C. A. English, J. Nucl. Mater. 205, 162 (1993).

[2] G. R. Odette and G. E. Lucas, JOM 53, 18 (2001).

[3] S. J. Zinkle and G. S. Was, Acta Mater. 61, 735 (2013)

[4] G. R. Odette and R. K. Nanstad, JOM 61, 17 (2009).

[5] P. B. Wells, T. Yamamoto, B. Miller, T. Milot, J. Cole, Y. Wu, and G. R. Odette, Acta Mater. 80, 205 (2014).

[6] D. Bacon, Y. Osetsky, and D. Rodney, Dislocation in Solids 88, 1 (2009).

[7] E. D. Eason, G. R. Odette, R. K. Nanstad, and T. Yamamoto, J. Nucl. Mater. 433, 240 (2013).

[8] C. L. Liu, G. R. Odette, B. D. Wirth, and G. E. Lucas, Mater. Sci. Eng. A 238, 202 (1997).

[9] E. Vincent, C. Becquart, C. Pareige, P. Pareige, and C. Domain, J. Nucl. Mater. 373, 387 (2008).

[10] F. Soisson, C. Becquart, N. Castin, C. Domain, L. Malerba, and E. Vincent, J. Nucl. Mater. 406, 55 (2010).

[11] D. Molnar, R. Mukherjee, A. Choudhury, A. Mora, P. Binkele, M. Selzer, B. Nestler, and S. Schmauder, Acta Mater. 60, 6961 (2012).

[12] S. R. Goodman, S. S. Brenner, and J. R. Low, Metall. Trans. 4, 2363 (1973).

[13] M. H. Mathon, A. Barbu, F. Dunstetter, F. Maury, N. Lorenzelli, and C. H. de Novion, J. Nucl. Mater. 245, 224 (1997).

[14] B. Radiguet, A. Barbu, and P. Pareige, J. Nucl. Mater. 360, 104 (2007).

[15] E. Meslin, M. Lambrecht, M. Hernandez-Mayoral, F. Bergner, L. Malerba, P. Pareige, B. Radiguet, A. Barbu, D. GomezBriceno, A. Ulbricht, and A. Almazouzi, J. Nucl. Mater. 406, 73 (2010).

[16] J. M. Hyde, G. Sha, E. A. Marquis, A. Morley, K. B.Wilford, and T. J. Williams, Ultramicroscopy 111, 664 (2011).

[17] E. Vincent, C. S. Becquart, and C. Domain, J. Nucl. Mater. 351, 88 (2006).

[18] E. Vincent, C. Becquart, and C. Domain, J. Nucl. Mater. 382, 154 (2008).

[19] G. R. Odette and B. D. Wirth, J. Nucl. Mater. 251, 157 (1997).

[20] R. Ngayam-Happy, C. S. Becquart, and C. Domain, J. Nucl. Mater. 440, 143 (2013).

[21] D. Kulikov, L. Malerba, and M. Hou, Phil. Mag. 86, 141 (2006).

[22] A. Al-Motasem, M. Posselt, F. Bergner, and U. Birkenheuer, J. Nucl. Mater. 414, 161 (2011).

[23] J. Marian, B. D. Wirth, A. Caro, B. Sadigh, G. R. Odette, J. M. Perlado, , and T. D. de la Rubia, Phys. Rev. B 65, $144102(2002)$.

[24] R. Pasianot and L. Malerba, J. Nucl. Mater. 360, 118 (2007).

[25] M. Mendelev, A. Han, D. Srolovitz, G. Ackland, D. Sun, and M. Asta, Phil. Mag. A 83, 3977 (2003).

[26] Y. Mishin, M. J. Mehl, D. A. Papaconstantopoulos, A. F. Voter, and J. D. Kress, Phys. Rev. B 63, 224106 (2001).

[27] C. C. Fu and F. Willaime, Phys. Rev. Lett. 92, 175503 (2004).

[28] J. P. Hirth and J. Lothe, Theory of Dislocations (McGraw-Hill, New York, 1968).

[29] D. Terentyev, X. He, E. Zhurkin, and A. Bakaev, J. Nucl. Mater. 408, 161 (2011).

[30] E. E. Zhurkin, D. Terentyev, M. Hou, L. Malerba, and G. Bonny, J. Nucl. Mater. 417, 1082 (2011).

[31] S. Plimpton, J. Comp. Phys. 117, 1 (1995).

[32] J. Li, Modelling Simul. Mater. Sci. Eng. 11, 173 (2003).

[33] Y. Nagai, T. Chiba, Z. Tang, T. Akahane, T. Kanai, M. Hasegawa, M. Takenaka, and E. Kuramoto, Phys. Rev. Lett. 87, $176402(2002)$.

[34] J. Marian, B. D. Wirth, J. M. Perlado, G. R. Odette, and T. D. de la Rubia, Phys. Rev. B 64, 094303 (2001).

[35] M. Ludwig, D. Farkas, D. Pedraza, and S. Schmauder, Modelling Simul. Mater. Sci. Eng. 6, 19 (1998).

[36] G. J. Ackland, D. J. Bacon, A. F. Calder, and T. Harry, Philos. Mag. A 75, 713 (1997).

[37] C. Domain and C. S. Becquart, Phys. Rev. B 65, 24103 (2002).

[38] C. S. Becquart and C. Domain, Nucl. Instrum. and Meth. B 86, 141 (2003).

[39] F. Soisson and C. C. Fu, Phys. Rev. B 76, 214102 (2007).

[40] O. I. Gorbatov, P. A. Korzhavyi, A. V. Ruban, B. Johansson, and Y. Gornostyrev, J. Nucl. Mater. 419, 248 (2011).

[41] P. Olsson, T. P. C. Klaver, and C. Domain, Phys. Rev. B 81, 054102 (2010).

[42] T. Ohnuma, N. Soneda, and M. Iwasawa, Acta Mater. 57, 5947 (2009).

[43] E. Vincent, C. S. Besquart, and C. Domain, J. Nucl. Mater. 359, 227 (2006).

[44] A. N. Stroh, Phil. Mag. 3, 625 (1958).

[45] R. G. A. Veiga, M. Perez, C. S. Becquart, C. Domain, and S. Garruchet, Phys. Rev. B 82, 054103 (2008).

[46] X. Y. Liu, J. Wang, and S. B. Biner, Modelling Simul. Mater. Sci. Eng. 16, 045002 (2008).

[47] L. Malerba, M. C. Marinica, N. Anento, C. Bjrkas, H. Nguyen, C. Domain, F. Djurabekova, P. Olsson, K. Nordlund, A. Serra, D. Terentyev, F. Willaime, and C. S. Becquart, J. Nucl. Mater. 406, 19 (2010). 
[48] N. I. Medvedeva, A. S. Murthy, V. L. Richards, D. C. V. Aken, and J. E. Medvedeva, J. Mater. Sci 48, 1377 (2013).

[49] Z. Yao, M. Hernandez-Mayoral, M. L. Jenkins, and M. A. Kirk, Phil. Mag. 88, 2851 (2008).

[50] B. Yao, D. J. Edwards, and R. J. Kurtz, J. Nucl. Mater. 434, 402 (2013).

[51] M. K. Miller and K. F. Russel, J. Nucl. Mater. 371, 145 (2007).

[52] W. Ostwald, Lehrbuch der Allgemeinen Chemie 2, 1 (1896)

[53] E. Meslin, B. Radiguet, and M. Loyer-Prost, Acta Mater. 61, 6246 (2013).

[54] G. Bonny, D. Terentyev, E. E. Zhurkin, and L. Malerba, J. Nucl. Mater. 452, 486 (2014). 
